\title{
Compactifying M-theory to four dimensions
}

\author{
Katrin Becker \\ California Institute of Technology 452-48, Pasadena, CA 91125 \\ CIT-USC, Center for Theoretical Physics, USA \\ E-mail: "beckerk̄otheory.caitech.èü

\section{Melanie Becker} \\ Department of Physics, University of Maryland, College Park \\ MD 20742-4111, USA \\ E-mail: 'melaniebophysics.umd.edù
}

Abstract: We consider compactifications of M-theory to four-dimensional Minkowski space on seven-dimensional non-compact manifolds. These compactifications include a warp factor which is non constant due to the presence of sources coming from five-branes wrapping two-dimensional submanifolds of the internal seven-dimensional space. We derive the expression for the field strengths and consider an explicit example of this general class of solutions.

KEYwords: 'M-Theory,-Supererstring Vacuà: 


\section{Contents}

ii. Introduction

2. M-theory compactifications to $d=4$ Minkowski space

i2.1' Compact seven-manifolds

12.2 Non-compact seven-manifolds

3. The Fayyazzudin-Smith manifold

望. Conclusions and outlook

'A- Useful formulas and conventions

\section{Introduction}

Recently warped compactifications of M-theory and $\mathcal{F}$-theory have become a fascinating arena of research because among other things it was suggested in 鄑 that these compactifications may provide us with a string theoretic realization of the RandallSundrum (RS) scenario [2, drum argued that our four-dimensional world could be described as the world-volume of a "threebrane". In the five-dimensional example suggested by Randall and Sundrum gravity is localized on the four-dimensional "brane" even if the fifth dimension is infinitely extended. This was only possible because of an exponential warp factor in the metric.

Warped compactifications have been known in string theory for a long time (see

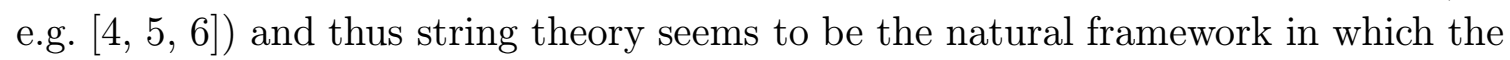
RS-scenario could be realized. Indeed, it was suggested in [i] that compactifications of $\mathcal{F}$-theory on an elliptically fibered Calabi-Yau four-fold 㓷 provide a realization of the RS-scenario and the consistency conditions following from supersymmetry can be obtained from the M-theory compactifications on four-folds considered in [商].

Warped compactifications play also an important role in the description of confining supersymmetric gauge theories and ultimately in the description of QCD. This is because there is a close relation between warped compactifications and Ramond- 
Ramond backgrounds in string theory. Confining gauge theories can be realized, for example, as perturbations by 3 -form flux of type-IIB string theory on $A d S_{5} \times S_{5}$. In this case the resulting supergravity theory has a naked singularity and it was shown by Polchinski and Strassler [9.9] that this singularity actually corresponds to an expanded brane source. The 3-form flux of the supergravity theory corresponds to a perturbation of the $\mathcal{N}=4$ gauge theory by mass terms and the resulting gauge

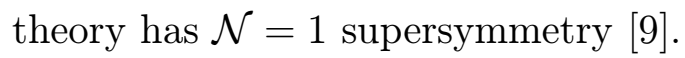

Non-perturbatively, $\mathcal{N}=1$ supersymmetric gauge theories can be realized by placing D3-branes at conical singularities of a Ricci-flat six-dimensional cone whose base manifold is a five-dimensional Einstein space $X_{5}$. On the supergravity side one considers type-IIB theory on $A d S_{5} \times X_{5}$ and this is dual to the world-volume theory of the D3-branes at the singularity. In case that one considers D3-branes on the conifold $\left[\begin{array}{l}1 \\ 1\end{array} \overline{0}\right]$, for example, one would obtain on the worldvolume of the D3-branes a gauge theory with $\mathrm{SU}(N) \times \mathrm{SU}(N)$ gauge group. Besides considering D3-branes it is also possible to consider D5-branes wrapped on collapsed 2-cycles at the singularity [i] ${ }_{1}^{1} \overline{1}_{1}$. This has the effect that the D3-brane charge eventually becomes negative and the supergravity metric becomes singular. It was argued by Klebanov and Strassler [12] that this naked singularity of the metric gets resolved in terms of a warped deformed conifold which is completely non singular. It was realized recently in [1] Klebanov-Strassler model can be obtained as a special case of the solutions derived

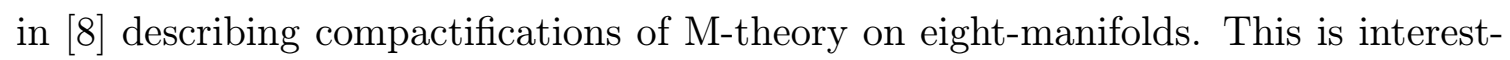
ing and one may wonder if there is a similar connection between the models considered in [8] model. This would be useful to derive the exact solution of the model considered in [יר:

In this paper we would like to broaden the class of theories that admit nonvanishing tensor fields. We would like to consider compactifications of M-theory to four-dimensional Minkowski space on seven-dimensional non-compact manifolds. These compactifications will involve a warp factor and we will see that we are able to construct solutions with non-vanishing expectation values for the tensor fields and a non-constant warp factor while supersymmetry is being preserved.

It has been known for some time that supersymmetry requires that compactifications of eleven-dimensional supergravity to four-dimensional Minkowski space have vanishing expectation values for the 4-form field strength and a constant warp factor if the internal manifold is compact and no sources are being considered $\left[\overline{1} \overline{1} \overline{5_{1}}, 1, \overline{6}_{1}^{1}\right]$.

But the argument presented in the previous two papers fails if the internal manifold is non compact or sources are considered as we will see in this paper. We shall see that in this case one is able to preserve supersymmetry and obtain non-vanishing expectation values for tensor fields and a non-constant warp factor at the same time. The sources that we shall be interested in originate from M-theory five-branes wrap- 
ping two-dimensional submanifolds of the internal seven-dimensional space. This has the consequence that the internal space is non compact. We will derive the explicit form for the field strength that follows from supersymmetry.

An interesting example of the general class of solutions that we will find here was considered in [1] dimensional Kähler manifold times a three torus. In this example the M-theory five-branes are wrapped on 2-cycles of the four-dimensional Kähler manifold. We will show explicitly that this example solves the equation for the field strength obtained herein.

This paper is organized as follows. In section ${ }_{2}^{2}$ w we consider compactifications of M-theory to four-dimensional Minkowski space on non-compact seven-manifolds. In section $2 .{ }_{1}^{1}$ we review the argument which shows why the field strengths are vanishing and the warp factor is constant for compactifications of M-theory on compact sevenmanifolds. In section $2 .{ }_{2}^{1}$ we consider non-compact seven-manifolds where sources coming from five-branes wrapping two-dimensional submanifolds of the internal space are taken into account. We derive the expression for the field strength in four dimensions that follows from supersymmetry and show that the warp factor is non constant due to the presence of these sources. In section

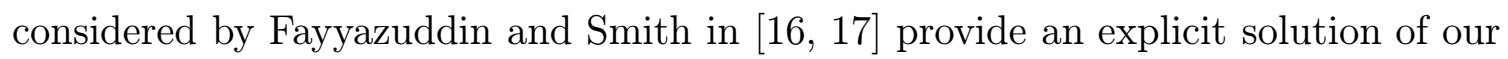

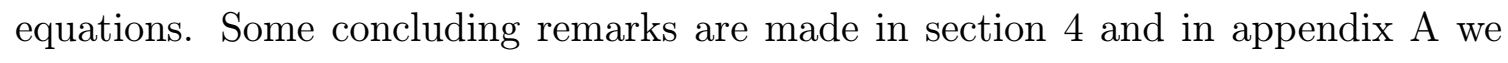
list some relevant formulas.

\section{M-theory compactifications to $d=4$ Minkowski space}

In this section we would like to consider compactifications of eleven-dimensional supergravity on non-compact seven-manifolds and derive the explicit form of the 4 -form field strength that follows from supersymmetry. ${ }^{1}$

\subsection{Compact seven-manifolds}

Let us first start by reviewing the argument that leads to the conclusion that supersymmetry implies that the warp factor is constant and thus the field strengths

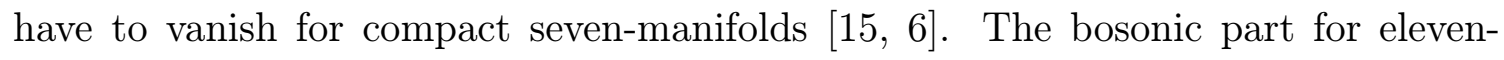
dimensional supergravity lagrangian contains a 3 -form $C$ with field strength $F$ and the dual 7 -form $\star F$

$$
\begin{aligned}
\mathcal{L}=\frac{1}{\kappa^{2}} \int d^{11} x \sqrt{g} & -\frac{1}{2} R-\frac{1}{48} F_{I J K L} F^{I J K L}- \\
& \left.-\frac{\sqrt{2}}{3456} \epsilon^{I_{1} I_{2} \cdots I_{11}} C_{I_{1} I_{2} I_{3}} F_{I_{4} \cdots I_{7}} F_{I_{8} \cdots I_{11}}\right) .
\end{aligned}
$$

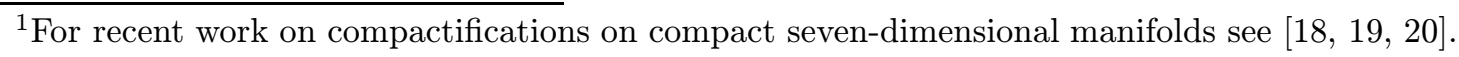


The complete action is invariant under the supersymmetry transformations

$$
\begin{aligned}
\delta e_{I}{ }^{m} & =\frac{1}{2} \bar{\eta} \Gamma^{m} \Psi_{I}, \\
\delta C_{I J K} & =-\frac{\sqrt{2}}{8} \bar{\eta} \Gamma_{[I J} \Psi_{k]}, \\
\delta \Psi_{M} & =\nabla_{M} \eta+\frac{\sqrt{2}}{288}\left(\Gamma_{M}^{P Q R S}-8 \delta_{M}^{P} \Gamma^{Q R S}\right) F_{P Q R S} \eta .
\end{aligned}
$$

The Einstein equation following from $\left(\underline{2}, \overline{1}_{1}\right)$ takes the form

$$
R_{M N}-\frac{1}{2} g_{M N} R+T_{M N}=0
$$

where $T_{M N}$ is the energy-momentum tensor of the 4-form field strength $F$ given by

$$
T_{M N}=4 F_{M P Q R} F_{N}^{P Q R}-\frac{1}{2} g_{M N} F_{P Q R S} F^{P Q R S} .
$$

In eleven dimensions one could in principle have membranes and five-branes that couple to the action ( $\left.\overline{2}_{2} \overline{1}_{1}^{\prime}\right)$ and appear as sources in the equation of motion for $C$ and Bianchi identify, respectively. Ignoring (for the moment) the presence of these sources $C$ satisfies the equation of motion

$$
d \star F=-\frac{1}{2} F \wedge F
$$

and the Bianchi identity

$$
d F=0
$$

We now would like to consider a line element of the form:

$$
d s^{2}=g_{M N} d x^{M} d x^{N}=\Delta^{-1}(y) \eta_{\mu \nu} d x^{\mu} d x^{\nu}+g_{m n}(y) d y^{m} d y^{n} .
$$

Here $\Delta(y)$ is the warp factor that because of Poincaré invariance depends only on the coordinates of the internal manifold, $g_{m n}$ is the metric of the internal sevendimensional space and $\eta_{\mu \nu}$ is the four-dimensional Minkowski space metric.

In compactifications with a maximally symmetric four-dimensional space-time the non-vanishing components of the 4 -form field strength are

$$
\begin{aligned}
& F_{m n p q}, \\
& F_{\mu \nu \rho \sigma}=f \epsilon_{\mu \nu \rho \sigma} .
\end{aligned}
$$

Here $f$ is arbitrary and will be determined later on and $\epsilon_{\mu \nu \rho \sigma}$ is the antisymmetric tensor of four-dimensional Minkowski space. Taking into account the decom-

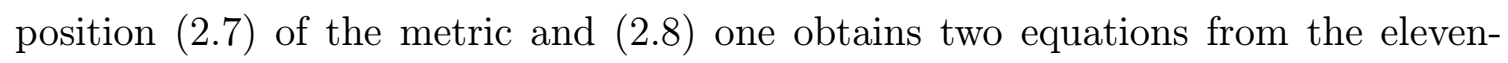
dimensional Einstein equation ( $(2 . \overline{3})$, one for the external component (i.e. where the 
Ricci-tensor has four-dimensional Minkowski indices) and one for the internal component (where the Ricci-tensor has seven-dimensional indices). The equation for the external component leads to the following equation:

$$
\nabla^{m}\left(\Delta^{-3} \partial_{m} \Delta\right)=-\frac{2}{3} \Delta^{-2}\left(F^{2}+48 \Delta^{4} f^{2}\right)
$$

where the covariant derivative involves the Christoffel connection as usual. Using Stokes theorem we see that the integral of the l.h.s. of this equation over a compact manifold vanishes. To conclude that all the expectation values of $F$ actually vanish one uses the observation that the r.h.s. of eq. ( $\left(2 . \overline{9}_{1}^{\prime}\right)$ is negative. Therefore all the components of $F$ must vanish

$$
F_{P Q R S}=0
$$

Going back to eq. (2.2.9.

$$
\nabla^{m}\left(\Delta^{-3} \partial_{m} \Delta\right)=0
$$

whose only solution on a compact manifold is

$$
\Delta=\text { const }
$$

Therefore, we recover the conventional supergravity compactifications in which no warp factor was taken into account.

\subsection{Non-compact seven-manifolds}

However, the argument of the previous section fails in a rather interesting way for non-compact internal seven-manifolds where, for example, M-theory five-branes that wrap cycles of the seven-manifold are taken into account. For non-compact manifolds the integral over the 1.h.s. of $\left({ }_{2} \overline{2}_{2} \overline{9}_{1}^{\prime}\right)$ is not equal to zero because of boundary terms. Furthermore, external sources modify the r.h.s. of this equation because these sources contribute to the energy momentum tensor (2.2.4) and therefore to the Einstein equation $\left(2 . \overline{2} \cdot \overline{3}^{\prime}\right)$. Very generally, we will consider seven-manifolds having a two-dimensional submanifold on which the five-brane can be wrapped. The five-brane worldvolume is of the form $M_{4} \times \Sigma$, where $M_{4}$ is four-dimensional Minkowski space and $\Sigma$ the twodimensional submanifold of the seven-dimensional internal space. As we will see in the following, in this situation it is possible to find non-vanishing expectation values for antisymmetric tensor fields with unbroken supersymmetry and a non-constant warp factor.

To find these solutions we will perform a similar analysis as in [i్s] but now for non-compact seven-manifolds. Unbroken supersymmetry requires that the transfor-

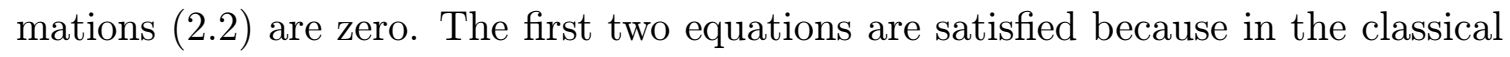


background the gravitino vanishes. We therefore only have to consider the super-

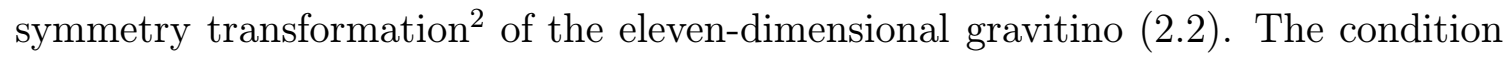
for unbroken supersymmetry

$$
\delta \Psi_{M}=0,
$$

will be decomposed into the external and internal components as follows:

$$
\begin{aligned}
\left(\nabla_{\mu}+\Delta^{-1 / 2} \gamma_{\mu} T\right) \eta & =0 \\
\left(\nabla_{m}+O_{m}\right) \eta & =0
\end{aligned}
$$

Here we have defined the quantities

$$
\begin{aligned}
T & =\frac{\sqrt{2}}{288}\left(F-i f \Delta^{2} \gamma_{5}-36 \sqrt{2} \gamma_{5} \not \partial \log \Delta\right), \\
O_{m} & =\frac{\sqrt{2}}{288}\left(\frac{1}{2} i f \gamma_{m}+\gamma_{5}\left(\gamma_{m} F-12 F_{m}\right)\right) .
\end{aligned}
$$

Furthermore, we have used the notation

$$
\begin{aligned}
F & =\gamma^{p q r s} F_{p q r s}, \\
F_{m} & =\gamma^{p q r} F_{m p q r},
\end{aligned}
$$

and $\not \partial=\gamma^{m} \partial_{m}$. The eleven-dimensional gamma matrices have been decomposed into two sets of mutually commuting gamma matrices according to

$$
\begin{gathered}
\Gamma_{\mu}=\gamma_{\mu} \otimes 1, \\
\Gamma_{m}=\gamma_{5} \otimes \gamma_{m},
\end{gathered}
$$

which is appropriate for an $11=4+7$ split. Furthermore, we have chosen our gamma matrices to be hermitean and $\gamma_{5}=\gamma_{1} \gamma_{2} \gamma_{3} \gamma_{4}$ satisfies $\left(\gamma_{5}\right)^{2}=1$. We will decompose the eleven-dimensional spinor $\eta$ according to

$$
\eta=\epsilon \otimes \xi
$$

where $\epsilon$ is a four-dimensional anticommuting spinor, while $\xi$ is a commuting sevendimensional spinor. Without loss of generality we will consider $\epsilon$ to be a positive chirality spinor $\gamma_{5} \epsilon=\epsilon$.

Since we are considering compactifications to four-dimensional Minkowski space we set

$$
\nabla_{\mu} \epsilon=0
$$

which for maximally symmetric four-dimensional space-time implies that the external space-time is flat Minkowski. Therefore, from the external component of the gravitino transformation (i.e. the first equation in $\left(2 \underline{2}_{-1} \overline{4}_{1}\right)$ ) one obtains the expression

$$
\left(F-i f \Delta^{2} \gamma_{5}-36 \sqrt{2} \gamma_{5} \not \partial \log \Delta\right) \eta=0 .
$$

\footnotetext{
${ }^{2}$ We will be following the notation and conventions of [6]"
} 


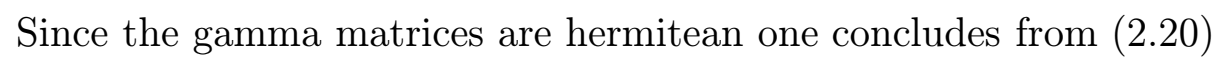

$$
f=0
$$

Therefore, unbroken supersymmetry does not allow external components $F_{\mu \nu \rho \sigma}$ for compactifications to four-dimensional Minkowski space independently if the internal manifold is compact or not. However, the situation is different for the internal components $F_{m n p q}$ which are now constrained to satisfy

$$
F \xi=36 \sqrt{2} \not \partial \log \Delta \xi .
$$

Notice that for compactifications of M-theory on seven-dimensional manifolds the equations satisfied by the field strengths are rather different than for compactifications of M-theory on eight-manifolds considered in [i] internal components of $F$ satisfy the equation

$$
F \xi=0
$$

rather than $\left(2.222_{1}^{1}\right)$. Furthermore it was found in [8] that the external component of $F$ could be expressed in terms of a derivative of the warp factor rather than having to vanish as in (12.2. $\left.\overline{1}_{1}^{\prime}\right)$.

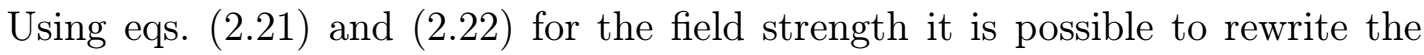
supersymmetry transformations of the gravitino ( $\overline{2}-14)$ in the following form:

$$
\begin{aligned}
\tilde{\nabla}_{m} \tilde{\xi}-\frac{\sqrt{2}}{24} \Delta^{3 / 2} F_{m p q r} \tilde{\gamma}^{p q r} \tilde{\xi} & =0, \\
F_{p q r s} \tilde{\gamma}^{p q r s} \tilde{\xi} & =36 \sqrt{2} \Delta^{-5 / 2} \partial_{a} \Delta \tilde{\gamma}^{a} \tilde{\xi}
\end{aligned}
$$

Here we have introduced a new metric $\tilde{g}_{m n}$ which is related to the metric $g_{m n}$ appearing in $\left(2 . \bar{z}_{1}\right)$ by a rescaling with the warp factor

$$
\tilde{g}_{m n}=\Delta g_{m n}
$$

The gamma matrices are rescaled accordingly $\tilde{\gamma}_{m}=\Delta^{1 / 2} \gamma_{m}$. We have also rescaled the seven-dimensional spinor $\xi$

$$
\tilde{\xi}=\Delta^{1 / 4} \xi,
$$

and used an identity relating covariant derivatives of spinors with respect to conformally transformed metrics that we have included in the appendix. The first relation in $(\overline{2} . \overline{2})$ guarantees that we can find a spinor whose norm is covariantly constant

$$
\tilde{\nabla}_{m}\left(\tilde{\xi}^{\dagger} \tilde{\xi}\right)=0
$$

and therefore we can choose the normalization

$$
\tilde{\xi}^{\dagger} \tilde{\xi}=1
$$


In terms of this spinor we can define a 2-form as

$$
\tilde{\omega}_{a b}=i \Delta^{-3} \tilde{\xi}^{\dagger} \tilde{\gamma}_{a b} \tilde{\xi}
$$

where we have introduced the warp factor for convenience. We shall see in a moment that the tensor field can be expressed in terms of this 2-form. In general, seven-dimensional manifolds are not characterized by 2 -forms. So for example, a $G_{2}$ holonomy manifold is characterized by a 3 -form $\Phi$ and it's Hodge dual 4 -form $\star \Phi$ and not by a 2 -form. But we can still expect to find seven-dimensional manifolds with non-vanishing 2-forms in special cases. Precisely these manifolds will be the interesting ones for which the field strengths have non-vanishing expectation values and the warp factor is non trivial. In the next section we will consider a seven-manifold that is a warped product of a four-dimensional Kähler manifold times a 3-torus. In this case the above 2-form is related to the Kähler form of the four-dimensional Kähler manifold. This type of compactification was considered in [i $\left.\overline{6}_{-1}^{1}, 1 \overline{1}\right]$. In this section we will derive the explicit expression for the expectation value of the tensor field. A more detailed analysis of the properties of the background geometry will appear

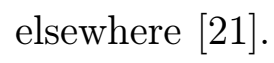

In eleven dimensions a 4 -form $F$ is dual to a 7 -form $\star F$. When compactifying on a seven-manifold to four-dimensional Minkowski space we can define a 3-form field strength $K$ in the following way:

$$
\star F=V_{4} \wedge K
$$

Here $V_{4}=d x^{0} \wedge d x^{1} \wedge d x^{2} \wedge d x^{3}$ is the constant volume element of the four-dimensional Minkowski space and $\star$ is the Hodge dual with respect to the eleven-dimensional metric $g_{M N}$. Using some gamma matrix identities that we list in the appendix and formulas ( $\left(2, \overline{2} \overline{4}_{1}^{\prime}\right)$ and $\left({ }_{2} \overline{2} . \overline{2} \overline{9}_{1}^{\prime}\right)$ it is possible to show that $K$ can be expressed through the derivatives of $\tilde{\omega}_{a b}$ in the following way:

$$
K_{a b c}=\frac{3}{\sqrt{2}} \tilde{\nabla}_{[a} \tilde{\omega}_{b c]} .
$$

To derive this equation it is useful to take the identity

$$
\tilde{\xi}^{\dagger}\left\{T, \tilde{\gamma}_{a b c}\right\} \tilde{\xi}=0
$$

into account. Here $T$ is defined as in $(2,1-15 i)$ with $f=0$. Remembering that the Christoffel connection $\Gamma_{M N}^{P}$ is symmetric in it's lower indices we see that $K$ can be written in the form

$$
K=\frac{1}{\sqrt{2}} d \tilde{\omega} .
$$

This is the general solution for $K$ that follows from supersymmetry. It is rather interesting that the $K$-field can be determined explicitly in terms of the 2-form ( $\left.\overline{2} \overline{2} . \overline{2} \bar{g}^{\prime}\right)$ rather than through a determining equation as for M-theory compactifications on 


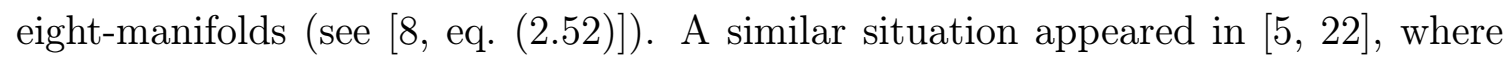

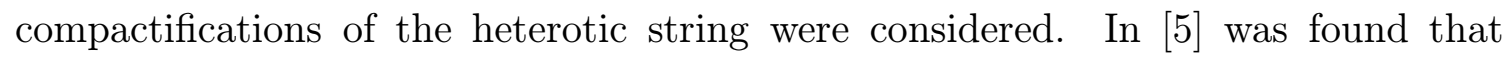
the $H$-field could be expressed in terms of a 2 -form (see eq. (2.17) of that paper), while the Yang-Mills field strength satisfies the Donaldson-Uhlenbeck-Yau equation, which is a determing equation similar as in [8]

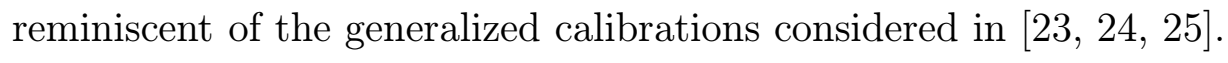

With the expression (12.33 $)$ for the $K$-field and ( above solution satisfies the eleven-dimensional five-brane Bianchi identity (or membrane equation of motion)

$$
d \star F=0 .
$$

Here we have to take into account that because the external components of $F$ vanish we have the condition $F \wedge F=0$. Since we are considering five-brane sources in eleven dimensions, the five-brane equation of motion (or membrane Bianchi identity) is no

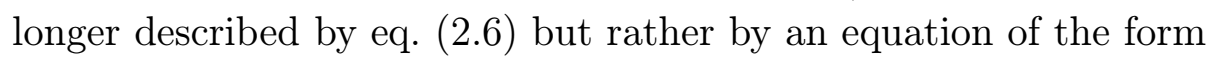

$$
d F=\delta
$$

Naively, the five-brane sources involve delta functions that are supported on the fivebrane worldvolume. These naive definition, however, leads to inconsistencies of the theory in the form of gravitational anomalies and a more careful analysis of the r.h.s.

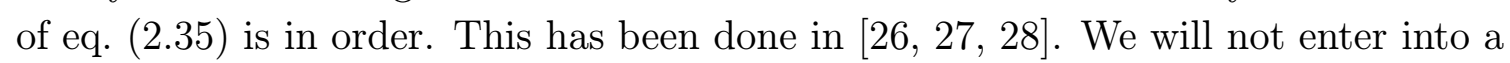
more detailed discussion of these issues here.

Finally, the five-brane sources will modify the r.h.s. of Einstein's equation (12..9') as the energy momentum tensor of the five-brane source has to be taken into account. Roughly, the external component of this equation takes the form:

$$
\nabla^{m}\left(\Delta^{-3} \partial_{m} \Delta\right)=-\frac{2}{3} \Delta^{-2} F^{2}+\delta
$$

where the delta functions come from the five-brane energy momentum tensor. Therefore, if sources are taken into account the warp factor no longer satisfies a Laplace equation on a compact manifold and the conclusions of section '2.

At this point we have determined the expectation value for the tensor field that follows from supersymmetry. Equation (2.36in is an equation for the warp factor, while $\left(2 . \overline{3} \overline{5}_{1}^{\prime}\right)$ is a determining equation for the 2 -form $\left(2 . \overline{2} \overline{9}_{1}^{\prime}\right)$. The non-vanishing component of the tensor field then follows from (2.31). The internal component of Einstein's equation will then provide the information on what the possible sevendimensional backgrounds are. A detailed analysis of the possible background geometries will appear elsewhere [2]1]. In the next section we shall consider a particular background geometry. 


\section{The Fayyazzudin-Smith manifold}

In this section we would like to consider a special seven-dimensional manifold which is a warped product of a four-dimensional Kähler manifold times a three-torus. We will consider five-brane sources and the five-brane wraps a 2-cycle of the four-dimensional Kähler space. This example of seven-manifold gives a supersymmetric compactification of M-theory and was considered by Fayyazuddin and Smith in [i] $\left.\overline{6}_{1}^{6}, 1 \overline{1}\right]$. In this case the 2-form $\tilde{\omega}$ appearing in $\left(\overline{2} . \overline{2} \bar{g}_{-1}^{\prime}\right)$ and $\left({ }_{2} . \overline{3} \overline{1}_{1}^{\prime}\right)$ arises naturally in terms of the Kähler form of the four-dimensional Kähler space.

The rescaled line element considered in this compactification is

$$
d \tilde{s}^{2}=\eta_{\mu \nu} d x^{\mu} d x^{\nu}+2 g_{m \bar{n}} d y^{m} d y^{\bar{n}}+\Delta^{3}(y) \delta_{\alpha \beta} d y^{\alpha} d y^{\beta}
$$

where $g_{m \bar{n}}$ is the metric of the four-dimensional Kähler space and $\delta_{\alpha \beta}$ is the metric of the three-torus. $g_{m \bar{n}}$ and $\Delta(y)$ are allowed to depend on both $y^{m}$ and $y^{\alpha}$.

The 2-form has the several components. The relevant one is the Kähler form of the four-dimensional manifold $\tilde{\omega}=\Delta^{-3} J$. Using (ㄴ.2. $\left.2 \overline{9}_{1}^{\prime}\right)$ we can compute $K$ and get

$$
K=\frac{1}{\sqrt{2}} d\left(\Delta^{-3} J\right)
$$

This is precisely the result obtained in the papers by Fayyazuddin and Smith (see e.g. [1] 1 i.j, eqs. (4)-(6)]) after dualizing and taking a rescaling of $F$ into account. There-

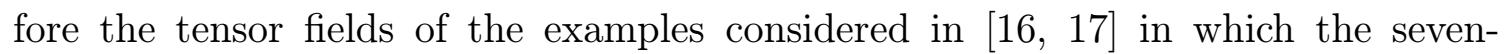
manifold is a warped product of a four-dimensional Kähler manifold times a threetorus provide an explicit example of the general class of solutions described in the previous section.

\section{Conclusions and outlook}

In this paper we have considered warped compactifications of M-theory on noncompact seven-manifolds to four-dimensional Minkowski space. Conventional compactifications of M-theory on compact seven-manifolds lead to vanishing expectation values for the tensor field and a constant warp factor if supersymmetry is imposed. However, this is not the case for the compactifications considered herein, where it is possible to preserve supersymmetry while the expectation value of the tensor fields are non vanishing and the warp factor is not constant. This is due to the presence of five-brane sources which wrap 2-cycles of the internal seven-dimensional manifold. We have computed the expression for the field strength following from supersymmetry explicitly. Furthermore, we have shown that the compactifications considered in [1] 10,1$]$ in provide a concrete example of our general solutions. 
It would certainly be interesting to see if further examples that solve (2.33i) could be found. In this paper we have seen how to obtain the form of tensor field once the background geometry is known. But supersymmetry can, of course, teach us more. A detailed analysis of the properties of the background geometry will appear elsewhere [2] $\left[\begin{array}{l}2 \\ 1\end{array}\right]$.

\section{Acknowledgments}

It is a pleasure to thank H. Verlinde and especially E. Witten for discussions and for bringing ref. [1] to our attention. We also would like to thank A. Strominger for discussions on warped compactifications some time ago. This work was supported by the U.S. Department of Energy under grant DE-FG03-92-ER40701.

\section{A. Useful formulas and conventions}

In this appendix we would like to collect a few useful formulas and we would like to explain our notation.

- The different types of indices that we use are

- $M, N, \ldots$ are eleven-dimensional indices

- $m, n, \ldots$ denote seven-dimensional indices

- $\mu, \nu, \ldots$ are the indices of the external space

- $n$-forms are defined with a factor $1 / n$ !. For example

$$
F=\frac{1}{4 !} F_{m n p q} d x^{m} \wedge d x^{n} \wedge d x^{p} \wedge d x^{q}
$$

- The gamma matrices $\Gamma_{M}$ are hermitean while $\Gamma_{0}$ is antihermitean. They satisfy

$$
\left\{\Gamma_{M}, \Gamma_{N}\right\}=2 g_{M N}
$$

- $\Gamma_{M_{1} \ldots M_{n}}$ is the antisymmetrized product of gamma matrices

$$
\Gamma_{M_{1} \cdots M_{n}}=\Gamma_{\left[M_{1} \cdots\right.} \Gamma_{\left.M_{n}\right]},
$$

where the square bracket implies a sum over $n$ ! terms with a $1 / n$ ! prefactor.

- Gamma matrix identities that are useful are

$$
\begin{aligned}
{\left[\gamma_{m}, \gamma^{r}\right] } & =2 \gamma_{m}^{r} \\
{\left[\gamma_{m n p}, \gamma^{r s}\right] } & =12 \delta_{\left[m^{[r} \gamma_{n p}\right]^{s]}} \\
\left\{\gamma_{m n p q}, \gamma^{r s t}\right\} & \left.=2 \gamma_{m n p q}{ }^{r s t}-72 \delta_{[m n}{ }^{[r s} \gamma_{p q]}\right]^{t]}
\end{aligned}
$$


- Our definition of Hodge $\star$ in $d$ dimensions is

$$
\star\left(d x^{m_{1}} \wedge \cdots \wedge d x^{m_{p}}\right)=\frac{|g|^{1 / 2}}{(d-p) !} \epsilon_{m_{p+1} \cdots m_{d}}^{m_{1} \cdots m_{p}} d x^{m_{p+1}} \wedge \cdots \wedge d x^{m_{d}},
$$

where

$$
\epsilon_{m_{1} m_{2} \cdots m_{d}}= \begin{cases}0 & \text { any two indices repeated } \\ +1 & \text { even permutation } \\ -1 & \text { odd permutation }\end{cases}
$$

- The identity which relates covariant derivatives of spinors with respect to conformally transformed metrics is

$$
\begin{aligned}
& \tilde{\nabla}_{M} \epsilon=\nabla_{M} \epsilon+\frac{1}{2} \Omega^{-1} \Gamma_{M}{ }^{N}\left(\nabla_{N} \Omega\right) \epsilon, \\
& \tilde{g}_{M N}=\Omega^{2} g_{M N} .
\end{aligned}
$$

\section{References}

[1] C.S. Chan, P.L. Paul and H. Verlinde, A note on warped string compactification, Nôncli: (1)

[2] L. Randall and R. Sundrum, A large mass hierarchy from a small extra dimension, Phys. Rev. Lett. 83 (1999) 3370 [hep-ph/9905221

[3] L. Randall and R. Sundrum, An alternative to compactification, $\stackrel{P}{\mathrm{P} h y s}$. Rev. Lett. $\mathbf{8} \overline{3}_{1}^{\prime}$

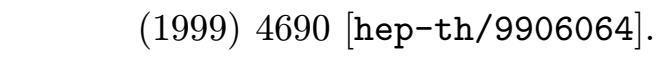

[4] B. de Wit, H. Nicolai and N.P. Warner, The embedding of gauged $N=8$ supergravity

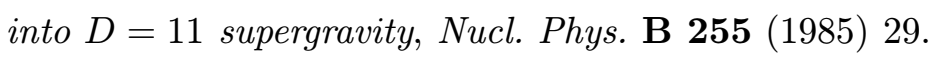

[5] A. Strominger, Superstrings with torsion, 1 Nucl. Phys. B $\mathbf{2} 7 \overline{4}(1 \overline{9} 86)$

[6] B. de Wit, D.J. Smit and N.D. H. Dass, Residual supersymmetry of compactified $D=10$ supergravity, iNucl. Phys. B $\mathbf{2} 8 \overline{3}-1987)-165$

[7] S. Sethi, C. Vafa and E. Witten, Constraints on low-dimensional string compactifications, iNucl. Phys. B 480 (1996) 213 [hep-th/9606122].

[8] K. Becker and M. Becker, M-theory on eight-manifolds, ${ }_{1}^{N}$ ucl. Phys. B $\overline{4} \overline{7} \overline{7}(1996)$ 1 $5 \overline{5}$ [hep-th/9605053'.

[9] J. Polchinski and M.J. Strassler, The string dual of a confining four-dimensional gauge theory, hep-th/0003136!

[10] I.R. Klebanov and E. Witten, Superconformal field theory on threebranes at a Calabi-

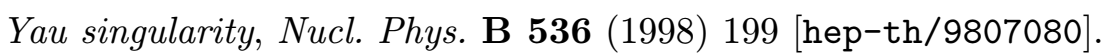

[11] I.R. Klebanov and A.A. Tseytlin, Gravity duals of supersymmetric $\mathrm{SU}(N) \times \mathrm{SU}(N+M)$ gauge theories, iNucl. Phys. B $\mathbf{5 7 8}(2000)$ 123i [hep-th/0002159.]. 
[12] I.R. Klebanov and M.J. Strassler, Supergravity and a confining gauge theory: duality

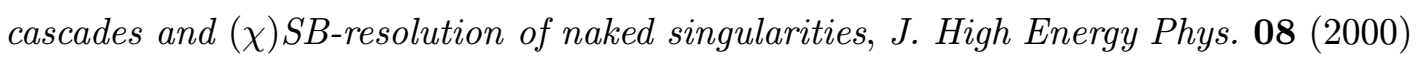

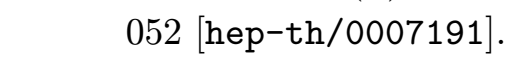

[13] M. Graña and J. Polchinski, Supersymmetric three-form flux perturbations on $A d S_{5}$,

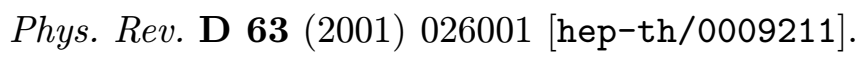

[14] S.S. Gubser, Supersymmetry and F-theory realization of the deformed conifold with three-form flux, hep-th/0010010'.

[15] P. Candelas and D.J. Raine, Spontaneous compactification and supersymmetry in $D=$ 11 supergravity, inucl. Phys. B 248 (1984) 415

[16] A. Fayyazuddin and D.J. Smith, Localized intersections of M5-branes and four-

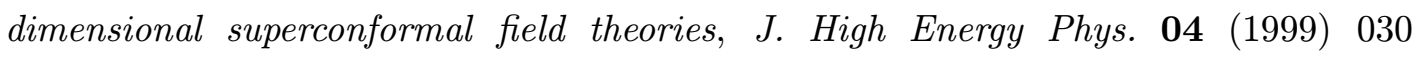
[hep-th/9902210i].

[17] B. Brinne, A. Fayyazuddin, S. Mukhopadhyay and D.J. Smith, Supergravity M5-branes wrapped on Riemann surfaces and their QFT duals, hep-th/0009047:

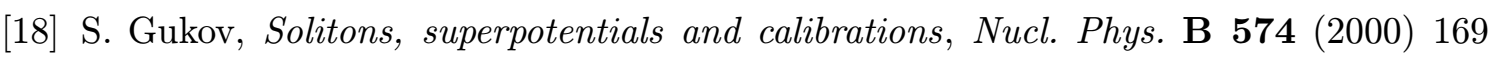
[hep-th/991101i].

[19] B.S. Acharya and B. Spence, Flux, supersymmetry and M-theory on 7-manifolds, hep-th/0007213.

[20] R. Hernandez, Calibrated geometries and non perturbative superpotentials in M-theory, hep-th/9912022

[21] K.Becker and M.Becker, work in progress.

[22] P. Candelas, G.T. Horowitz, A. Strominger and E. Witten, Vacuum configurations for

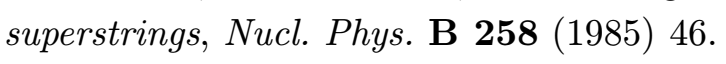

[23] J. Gutowski and G. Papadopoulos, AdS calibrations, 'Phys. Letzt. [hep-th/9902034].

[24] J. Gutowski, G. Papadopoulos and P.K. Townsend, Supersymmetry and generalized

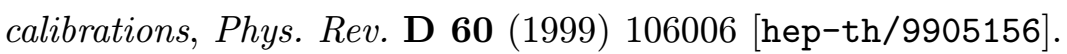

[25] H. Cho, M. Emam, D. Kastor and J. Traschen, Calibrations and fayyazuddin-smith

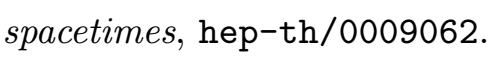

[26] E. Witten, Five-brane effective action in M-theory, J. Geom. Phys. 22 (1997) 103 [hep-th/9610234].

[27] D. Freed, J.A. Harvey, R. Minasian and G. Moore, Gravitational anomaly cancellation for M-theory fivebranes, 'Adv. Theor. Math. Phys 2 (1998) 601' [hep-th/9803205].

[28] K. Becker and M. Becker, Fivebrane gravitational anomalies, '№ucl. Phys. B $\mathbf{5} \overline{7} \overline{7}(200 \overline{0})$,

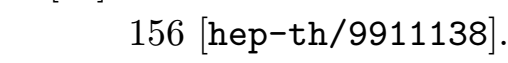

